\title{
Thermographic Study of Plant Response to Excessive Light
}

\author{
A. Orzechowska ${ }^{a, *}$, M. Trtílek ${ }^{b}$ And E. Niewiadomska ${ }^{c}$ \\ ${ }^{a}$ Faculty of Physics and Applied Computer Science, AGH University of Science and Technology, \\ al. A. Mickiewicza 30, 30-059 Kraków, Poland \\ ${ }^{b}$ Photon Systems Instruments, Drásov 470, 664 24 Drásov, Czech Republic \\ ${ }^{c}$ The F. Górski Institute of Plant Physiology, Polish Academy of Sciences, \\ Niezapominajek 21, 30-239 Kraków, Poland
}

Doi: 10.12693/APhysPolA.139.257 *e-mail: aleksandra.orzechowska@fis.agh.edu.pl

\begin{abstract}
Thermal imaging as a highly sensitive technique was used to study a rapid response of plants to excessive light associated with stomatal movement. This response was monitored according to timedependent changes in temperature over the leaf blades of plants exposed to photosynthetically active radiation. Two model organisms, Thellungiella salsuginea and Arabidopsis thaliana, which served as a control, were tested. A decreased stomata size in Thellungiella salsuginea resulted in an increased rosette temperature which was noticeable on the time-series thermograms as compared to controls. A primary response of plants to light revealed an exponential increase in the temperature time courses and resulted in a reduced thermal rise time for Thellungiella salsuginea relative to Arabidopsis thaliana seedlings. The study evaluates the use of thermal imaging to analyse stomatal aperture. This noninvasive method, in particular the evaluation of thermal kinetics and analysis of thermal time constants, can be useful for monitoring the initial response of plants to excess irradiation.
\end{abstract}

topics: infrared thermal imaging, leaf temperature, light-induced temperature kinetics, stomatal conductance

\section{Introduction}

Changes in leaf temperature have long been recognised as an indicator of plant stress. Excessive light and disturbance of evaporation are among the factors that affect this temperature to a large extent. In plants, transpiration takes place via special pores in leaf surface (stomata), which enable the diffusion of gases with the atmosphere. The main function of stomata is to balance the uptake of $\mathrm{CO}_{2}$, which is essential for photosynthesis, with the loss of water. Stomatal movement is regulated by environmental conditions such as light, $\mathrm{CO}_{2}$ and humidity. It is crucial in maintaining plant water status and photosynthetic rates depending on the current needs of the plant [1].

It is well known that leaf temperature depends on stomatal movement, with the temperature decreasing as the stomata open and as evaporation rates increase. The opposite, when stomata close, results in an increase in leaf temperature and a reduction in the rate of transpiration $[1,2]$. For that reason, thermal sensing of stomatal aperture is a precise indicator of light stress affecting plants [3-6]. Light, which is a necessary factor for conducting photosynthesis, induces the opening of stomata to enhance $\mathrm{CO}_{2}$ uptake. The opening response is achieved by coordinating light signaling, light-energy conversion, the transport of $\mathrm{K}^{+}$ions across the membrane and metabolic activity in specialized cells (called guard cells) in the stomata [2]. Distinct mechanisms underlie stomatal opening in response to different light wavelengths (blue and red) although it has been reported that green light also plays a vital role [7] in the inhibition of stomatal opening in order to prevent water loss in plants [8].

Thermal imaging provides a very powerful tool for the study of spatial and temporal variations in plant temperatures with many potential applications in plant physiology and ecology [9-11]. A general difficulty with thermal methods is that the temperature of plants may be affected by many environmental factors such as the surrounding temperature, humidity, air movements and radiation. The main advantage of thermal imaging, however, is that the method is non-invasive because infrared (IR) experiments do not require any physical contact with leaves. Moreover, when used in combination with other imaging techniques (e.g. fluorescence imaging) [5], IR imaging may serve to diagnose the initial symptoms of plant stress $[12,13]$.

Considering the benefits of thermography in plant research, the objective of this study was to investigate the applicability of thermal imaging to 
measuring the spatial distribution of the leaf temperature of two model organisms Arabidopsis thaliana (A. thaliana) and Thellungiella salsuginea (T. salsuginea) under intense illumination (high light HL). A. thaliana and its close relative T. salsuginea belong to the Brassicaceae family and T. salsuginea has become an extremophile model for abiotic stress tolerance studies $[14,15]$. The leaves of both species are characterized by simple geometry and fairly flat surface topography. However, the function and morphology of stomata in T. salsuginea is significantly different from those in $A$. thaliana [16]. Leaf temperature varies with a transpiration rate and it is dependent on stomatal conductance [17]. A smaller stomata size results in reduced stomatal conductance and transpiration flux in T. salsuginea [18]. This affects the diversity of temperature spatial distribution in rosettes of both species which is enhanced under HL treatment.

We aim to outline the light-induced temperature kinetics and consider thermal time constants so as to compare the response of $T$. salsuginea and A. thaliana to intense irradiation. In order to avoid the influence of any fluctuating environmental factors, the experiments were performed under fully controlled laboratory conditions.

\section{Materials and methods}

\subsection{Plant material and growth conditions}

For the purpose of this study, four-week old A. thaliana wild type (WT) and T. salsuginea plants were used. The seeds were obtained from the Nottingham Arabidopsis Stock Centre, UK. The plants were cultivated under fully controlled laboratory conditions in a phytotron chamber at a temperature of $23^{\circ} \mathrm{C}$, photoperiod of $16 / 8 \mathrm{~h}$ day/night, irradiance of $150 \mu \mathrm{mol}$ (photons) $\mathrm{m}^{-2} \mathrm{~s}^{-1}$ and in $65 \%$ relative humidity (FytoScope FS2700, PSI, Czech Republic). After the leaf rosettes had developed the plants were watered twice a week with the same volume of tap water. A. thaliana seedlings served as controls.

\subsection{Thermal experiments}

Thermal images were obtained using an uncooled thermal camera (Flir Systems Inc., USA) which operates within a spectral wavelength range of $7.5-14 \mu \mathrm{m}$ with an accuracy of $\pm 2 \%$ and noise equivalent temperature difference $>30 \mathrm{mK}$, equipped with the option to autocalibrate with the surrounding temperature. It produces a spatial resolution of $640 \times 480$ pixels. The emissivity of the IR camera was set to 0.95 which is within the range of emissivity of plant leaves [13]. Thermograms were acquired with a frequency of $50 \mathrm{~Hz}$ under illumination of $2000 \mu \mathrm{mol}$ (photons) $\mathrm{m}^{-2} \mathrm{~s}^{-1}$ at $5 \mathrm{~s}$ of intervals for $5 \mathrm{~min}$. All image processing and data export was undertaken using the software
IR-Visualizer (PSI, Czech Republic). The source of white light was a panel developed with lightemitting diodes (LEDs) with an automatic cooling option (SL 3500, PSI, Czech Republic) mounted on an adjustable stand at a fixed distance of $25 \mathrm{~cm}$ toward the leaf rosettes. The light intensity was monitored using a quantum meter equipped with optical detectors that provide high-accuracy photosynthetically active radiation (PAR) measurements in the range of 400-700 $\mathrm{nm}$ including colored LEDs (Apogee MQ-200). Thermal kinetics were evaluated on the basis of time series thermograms. To obtain the most accurate temperature for each desired reading, a minimum of three thermographs were taken. All thermal images were recorded for three $A$. thaliana seedlings juxtaposed with three T. salsuginea plants.

The goal was to assess the difference in temperature between $A$. thaliana and $T$. salsuginea leaves but not to determine the absolute leaf temperature. Only the leaves that had similar surface areas were taken for further analysis. IR measurements were carried out under controlled laboratory conditions, i.e., stable temperature and humidity with avoiding air movement and incident radiation.

\section{Results and discussion}

Infrared imaging is an accurate method of temperature spacial distribution. An exemplary thermal image that shows the differences in temperature between T. salsuginea and A. thaliana rosettes is presented in Fig. 1. One can note therefore the thermal distribution over the lamina without (Fig. 1a) and under light treatment (Fig. 1b).

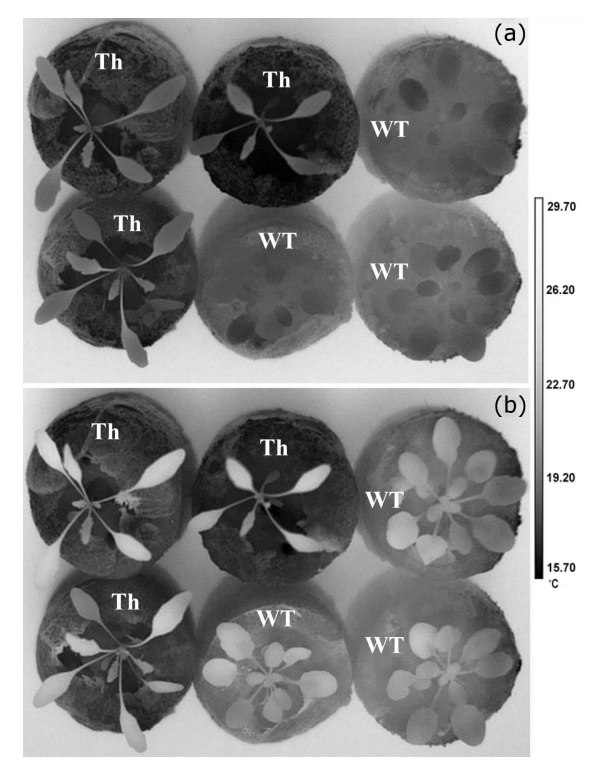

Fig. 1. Infrared thermographs of $T$. salsuginea (Th) plants relative to the control (WT) seedlings acquired before light onset (a) and under continuous irradiation of excessive light at an intensity of $2000 \mu \mathrm{mol}$ (photons) $\mathrm{m}^{-2} \mathrm{~s}^{-1}$ (b). 
One can see an obvious increase in leaf temperature caused by the action of HL (Fig. 1b), however, the dissimilarities in temperature between the species are noticeable even before the onset of light (Fig. 1a). Heterogeneity in temperature spacial distribution is also visible between the leaves in the subsequent stages of development within the rosette. Young leaves reveal a lower temperature when compared to older ones, although in the case of $T$. salsuginea this difference is more pronounced. The effect is enhanced under the light treatment.

Photosynthesis is very sensitive to intense illumination. PAR is indispensable for photosynthesis to take place, although an excess of it may decrease the photosynthetic efficiency or even damage photosynthetically active structures [19-21]. Since light onset influences the stomatal opening, it represents one of the main factors that have an impact on thermal stability in plant tissues. In our experiment, the significant increase in leaf temperature after the light onset (Fig. 1b) was caused mainly by the incoming light energy and inefficient evapotranspiration over the lamina [6] of the plants under investigation. Continuous illumination of seedlings and the light intensity $\left(2000 \mu \mathrm{mol}\right.$ (photons) $\mathrm{m}^{-2} \mathrm{~s}^{-1}$ ) used in the experiment was sufficient to reduce significantly the efficiency of the photosynthetic apparatus of the studied plants (data not shown).

The diversity of density and the area of stomata between $A$. thaliana and T. salsuginea influence the transpiration rate, thus the variety in leaf morphology between the two species also affects the temperature of the plant rosettes. A smaller stomata size in T. salsuginea [16] results in a reduced ability to evaporate which, in turn, leads to an increase in temperature. This is consistent with our results which show that in the case of $T$. salsuginea the temperature is significantly higher during the whole period of HL treatment (see Fig. 2).

An increased temperature can also be caused by a reduced stomata opening due to the influence of external stress factors, e.g. light. The lightinduced thermal courses were fitted using a monoexponential function

$$
T=T_{0}+T_{m}\left(1-\mathrm{e}^{-t / t_{m}}\right),
$$

where $T_{0}$ and $T_{m}$ are the amplitudes and $t_{m}$ is the thermal time constant. The analysis of the thermal rise time $t_{m}$ revealed that this value determined for $T$. salsuginea $\left(t_{m}=13.60 \pm 1.55 \mathrm{~s}\right)$ was approximately twofold decreased in comparison to A. thaliana $\left(t_{m}=26.70 \pm 3.50 \mathrm{~s}\right)$. These results correspond to those of the previous experiments performed on the mutant ost1-2 with an inability to regulate the stomata, resulting in enhanced stomatal conductance and transpiration rate, as reported in [5]. In this mutant, the ost 1 mutation contributed to the slowing down of thermal kinetics, unlike T. salsuginea kinetics which showed advanced temperature time course in comparison

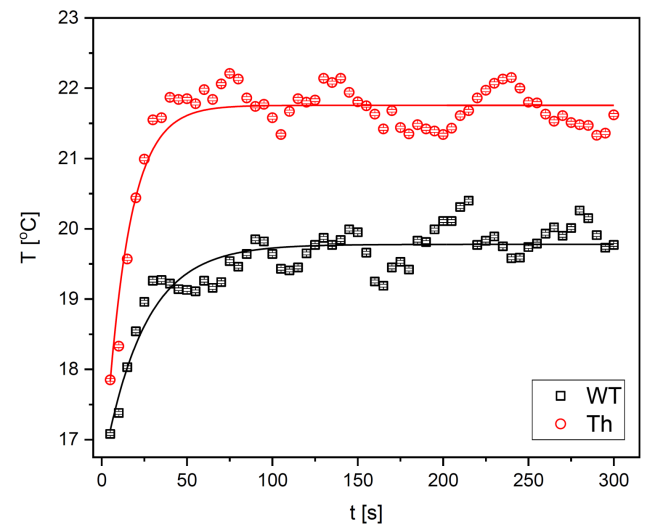

Fig. 2. Thermal kinetics evaluated under the action of excessive light over 5 min of illumination. Before the light onset plants were adapted to standard growth light. The exponential fit of the lightinduced temperature rise in the T. salsuginea ( $\mathrm{Th}$ - circular symbols) and A. thaliana (WT - square symbols) rosettes shows the primary response of plants to HL onset. For each thermogram of each of the T. salsuginea and A. thaliana plants, taken at the same time, the mean temperature of at least three randomly chosen spots was calculated. Temperature uncertainties are within the symbols.

to the controls. The decreased thermal time constant in T. salsuginea was caused by reduced evaporation, as compared to A. thaliana.

In our study, the thermal rise times were evaluated on the basis of IR images of the juxtaposed plants. Therefore, the time constants represent here the relative values between $T$. salsuginea and the control - A. thaliana. Unlike $[3,4,6]$, we did not use any material (e.g. paraffin wax) to cover the stomata in order not to limit or inhibit transpiration in the plants. We did not interfere with the physiological behavior of the stomata.

IR imaging is well suited to the study of the spatial diversity of stomatal conductance between plant leaves and the stomatal diversity ("patchy" effects) over lamina $[6,22]$. There are some reports showing a coupling between stomata in different areas of the leaf during transpiration [22, 23]. The patchiness in stomatal action is mainly due to variation in stomatal density which is dependent on leaf morphology [6] and hydraulic interactions within the stomatal net [24]. Our results in Fig. 2, which show the characteristic fluctuations in temperature during the course, may be associated with stomatal diversity. The oscillations in leaf temperature over the leaf blade may also be attributable to a plant strategy to protect itself against heat stress under excess light [25].

\section{Conclusions}

In plant studies, IR thermography has particular advantages for the quantitative analysis of spacial and dynamic physiological information. 
Applications of thermography in plant physiology also include the investigation of thermogenesis as well as the influence of external factors that have an impact on plant thermal stability.

In this study, non-invasive thermal imaging, in particular time series thermograms, provided an analysis of the initial response of plants to light excess associated with stomatal movement. Moreover, the determination of thermal time constants may serve to diagnose the symptoms of excessive light affecting plants in a dynamic environment.

\section{Acknowledgments}

This work was supported by the Faculty of Physics and Applied Computer Science, AGH University of Science and Technology (No. 16.16.220.842) within subventions from the Ministry of Science and Higher Education.

\section{References}

[1] J.S.A. Matthews, S. Vialet-Chabrand, T. Lawson, J. Exp. Bot. 71, 2253 (2020).

[2] K. Shimazaki, M. Doi, S.M. Assmann, T. Kinoshita, Annu. Rev. Plant Biol. 58, 219 (2007).

[3] P. Bajons, G. Klinger, V. Schlosser, Infrared Phys. Technol. 46, 429 (2005).

[4] R. Kana, I. Vass, Environ. Exp. Bot. 64, 90 (2008).

[5] A. Orzechowska, M. Trtílek, K. Tokarz, P. Rozpądek, Biochim. Biophys. Res. Commun. 533, 1129 (2020).

[6] S. Vialet-Chabrand, T. Lawson, J. Exp. Bot. 70, 2839 (2019).

[7] H.L. Smith, L. McAusland, E.H. Murchie, J. Exp. Bot. 682099 (2017).

[8] K. Aasamaa, P.J. Aphalo, Environ. Exp. Bot. 121, 94 (2016).

[9] S.A. Raza, G. Prince, J.P. Clarkson, N.M. Rajpoot, Plos ONE 10, 1 (2015).
[10] V. Sagan, M. Maimaitijiang, P. Sidike, et al., Remote Sens. 11, 1 (2019).

[11] M. Urrestarazu, Quant. Infr. Therm. J. 10, 1 (2013).

[12] M. Jafari, S. Minaei, N. Safaie, Infrared Phys. Techn. 85, I170 (2017).

[13] H.G. Jones, Adv. Bot. Res. 41, 107 (2004).

[14] M. Pilarska, M. Wiciarz, I. Jajic, M. Kozieradzka-Kiszkurno, P. Dobrev, R. Vankova, E. Niewiadomska, Front. Plant Sci. 7, 1 (2016).

[15] M. Wiciarz, B. Gubernator, J. Kruk, E. Niewiadomska, Physiol. Plant. 153, 467 (2014).

[16] F. Orsini, M.P. D'Urzo, G. Inan et al., J. Exp. Bot. 61, 3787 (2010).

[17] H.G. Jones, Agric. Forest Meteorol. 95, 135 (1999).

[18] P. Stepien, G.N. Johnson, Plant Physiol. 149, 1154 (2009).

[19] E.-M. Aro, I. Virgin, B. Andersson, Biochim. Biophys. Acta 1143, 113 (1993).

[20] R. Szymańska, I. Ślesak, A. Orzechowska, J. Kruk, Environ. Exp. Bot. 139, 165 (2017).

[21] E. Niewiadomska, K. Brueckner, M. Mulisch, J. Kruk, A. Orzechowska, M. Pilarska, R. Luchowski, W.I. Gruszecki, K. Krupinska, J. Plant Physiol. 223, 57 (2018).

[22] G. Prytz, C.M. Futsaether, A. Johnsson, New Phytol. 158, 249 (2003).

[23] K. Omasa, K. Takayama, Plant Cell Physiol. 44, 1290 (2003).

[24] T.N. Buckley, K.A. Mott, Plant Cell Environ. 23, 301 (2000).

[25] M. Quint, C. Delker, K.A. Franklin, P.A. Wigge, K.J. Halliday, M. van Zanten, Nat. Plants 2, 15190 (2016). 